\title{
Checklist para cirurgia cardíaca segura: revisão integrativa
}

\author{
Checklist for safe cardiac surgery: integrative review \\ Lista de verificación para una cirugía cardíaca segura: revisión integradora
}

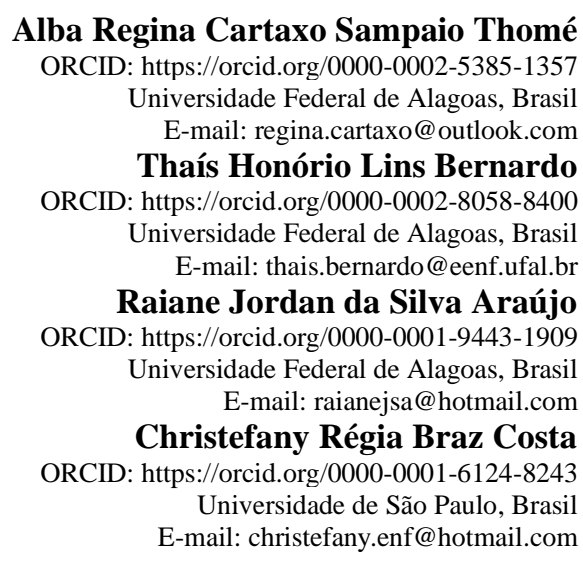

\begin{abstract}
Resumo
Objetivo: identificar na literatura checklist para cirurgia cardíaca segura. Metodologia: trata-se de uma revisão integrativa, composta por seis etapas, realizada nas bases Medline via Pubmed (Nacional Library of Medicine National Institutes of Health) Cinhal (Cumulative Index to Nursing and Allied Health Literature), Lilacs (Literatura Latino Americana e do Caribe em Ciências da Saúde), Scopus e Web of Scienc, entre setembro e outubro de 2020. Resultados: a busca resultou em oito artigos que apresentaram checklist para cirurgia cardíaca. Quanto à origem dos estudos a maior parte foi dos Estados Unidos da América (USA), 50\%, em relação aos profissionais que fizeram parte dos instrumentos foram incluídos anestesista, cirurgião, perfusionista, enfermeiro e instrumentador, sendo o profissional cirurgião presente e todas as listas e na fase cirúrgica a mais prevalente foi Time out. Conclusão: foi possível constatar na literatura a existência de checklist para cirurgia cardíaca segura com evidências que reúnem elementos de verificação para a segurança do paciente cardíaco.
\end{abstract}

Palavras-chave: Lista de checagem; Cirurgia torácica; Procedimentos cirúrgicos torácicos; Segurança do paciente; Cirurgia geral.

\begin{abstract}
Objective: to identify a checklist for safe cardiac surgery in the literature. Methodology: this is an integrative review, consisting of six stages, carried out in Medline databases via Pubmed (National Library of Medicine, National Institutes of Health), Cinhal (Cumulative Index to Nursing and Allied Health Literature), Lilacs (Latin American Literature and of the Caribbean in Health Sciences), Scopus and Web of Scienc, between September and October 2020. Results: the search resulted in eight articles that presented a checklist for cardiac surgery. As for the origin of the studies, most were from the United States of America (USA), 50\%, in relation to the professionals who were part of the instruments, anesthetist, surgeon, perfusionist, nurse and scrub nurse were included. lists and in the surgical phase the most prevalent was Time out. Conclusion: it was possible to verify in the literature the existence of a checklist for safe cardiac surgery with evidence that brings together verification elements for the safety of cardiac patients.
\end{abstract}

Keyword: Checklist; Thoracic surgery; Thoracic surgical procedures; Patient safety; General surgery.

\section{Resumen}

Objetivo: identificar una lista de verificación para la cirugía cardíaca segura en la literatura. Metodología: se trata de una revisión integradora, que consta de seis etapas, llevada a cabo en bases de datos de Medline vía Pubmed (Biblioteca Nacional de Medicina, Institutos Nacionales de Salud), Cinhal (Índice Acumulativo de Literatura de Enfermería y Afines en Salud), Lilacs (Literatura Latinoamericana y del Caribe en Ciencias de la Salud), Scopus y Web of Scienc, entre septiembre y octubre de 2020. Resultados: la búsqueda arrojó ocho artículos que presentaban una lista de verificación para cirugía cardíaca. En cuanto al origen de los estudios, la mayoría fueron de Estados Unidos de América (EE. UU.), 50\%, en relación a los profesionales que formaban parte de los instrumentos, se incluyeron anestesista, cirujano, perfusionista, enfermero y enfermero auxiliar. en la fase quirúrgica el más prevalente 
fue el Time out. Conclusión: se pudo verificar en la literatura la existencia de un checklist para cirugía cardíaca segura con evidencia que reúne elementos de verificación para la seguridad de los pacientes cardíacos.

Palabras clave: Lista de verificación; Cirugía torácica; Procedimientos quirúrgicos torácicos; Seguridad del paciente; Cirugía general.

\section{Introduçãa}

Mais de 200 milhões de cirurgias são realizados em todo o mundo a cada ano (World Health Organization, 2019). Relatórios recentes revelam que as taxas de eventos adversos para condições cirúrgicas permanecem inaceitavelmente altas apesar de várias iniciativas globais para a segurança do paciente na última década (Earnshaw \& Alderson, 2014).

O erro humano é inevitável, mas é mais provável que ocorra em condições estressantes, fadiga mental ou quando se desvia dos procedimentos convencionais (Kim et al., 2015; Liukka et al., 2020). Esses fatores são especialmente relevantes em cirurgia cardíaca, onde os casos são complexos, demorados e integram técnicas mais inovadoras (Hussain et al., 2016; Chan et al., 2019).

A cirurgia cardíaca dentre as cirurgias, é considerada uma das mais complexas e pode se beneficiar ao administrar parte dessa complexidade por meio do uso de listas de verificação (Konfirst, 2015). É um procedimento de alto risco intrínseco e um processo de várias etapas, com interrupções frequente do fluxo de trabalho, tornando-se a comunicação um desafio. E, como consequência, há grande interesse multidisciplinar em melhorar a cirurgia cardíaca (Ogden et al., 2016). Identificar as causas e desenvolver planos para minimizar ou eliminar os eventos adversos pode ajudar a estabelecer um sistema que garanta a segurança do paciente (Kim et al., 2015).

A Organização Mundial da Saúde (OMS) em 2009 lançou um checklist de segurança que inclui três etapas bem definidas, antes da indução anestésica (Sign in), antes da incisão cirúrgica (Time out), antes da saída de sala (Sign out) em que a equipe verifica itens de segurança e identifica possíveis riscos de erros, cujo objetivo é auxiliar de forma sistemática passos críticos de segurança (OMS, 2009; Russ et al, 2015). Esta lista de segurança não é adaptada para resolver problemas e fatores de risco de subespecialidades cirúrgicas, por isso a OMS recomenda a realização de ajustes a partir da lista primitiva (Kim et al., 2015).

A prática diária evidencia que, embora os instrumentos já utilizados incluam fatores gerais de risco, muitas vezes não incluem fatores específicos para as cirurgias cardíacas (Thomé et al., 2017). A partir disso faz-se necessário a identificação de checklist específico que atenda as reais necessidades da cirurgia cardíaca segura, subsidiando o cuidado de enfermagem e da equipe cirúrgica a promover melhoria na assistência. Nesse contexto, o estudo teve como objetivo identificar na literatura checklist para cirurgia cardíaca segura, tendo como base a seguinte pergunta de pesquisa: Quais checklist estão disponíveis na literatura para cirurgia cardíaca segura?

\section{Metodologia}

Trata-se de uma revisão integrativa (RI), composta por seis etapas: definição da pergunta de pesquisa, busca e seleção dos estudos primários, extração de dados dos estudos primários, avaliação crítica dos estudos primários, síntese dos resultados da revisão, apresentação da revisão (Mendes et al., 2019). Para a compreensão do conteúdo abordado foi elaborado as estratégias de busca dos estudos primários e foram combinadas conforme cada elemento da estratégia PICo (P População/Paciente/problema; I - Interesse; Co- Contexto) (Joanna Briggs Institute, 2014). O (P) Segurança do paciente (I) checklists (Co) cirurgia cardíaca.

A busca foi realizada entre Setembro e Outubro de 2020. As bases de dados selecionadas para a presente pesquisa foram: Medline via Pubmed (Nacional Library of Medicine National Institutes of Health) por meio de descritores em inglês Mesh (Medical Subject Headings), Cinhal (Cumulative Index to Nursing and Allied Health Literature) nos títulos (Headings), 
Lilacs (Literatura Latino Americana e do Caribe em Ciências da Saúde) pelo (Descs), Scopus e Web of Scienc com palavras chaves, conforme Quadro 1.

Quadro 1 - Estratégias de busca nas bases de dados. Maceió, AL, Brasil, 2020.

\begin{tabular}{|c|c|c|c|}
\hline \multicolumn{4}{|c|}{ Pesquisa - Medline via Pubmed } \\
\hline 1 & & Checklist & Mesh \\
\hline 2 & $A N D$ & Thoracic Surgery & Mesh \\
\hline 3 & $O R$ & Thoracic Surgical Procedures & Mesh \\
\hline \multicolumn{3}{|c|}{$\begin{array}{l}\text { Estratégia - ("Checklist"[Mesh]) AND ("Thoracic Surgery"[Mesh] OR "Thoracic } \\
\text { Surgical Procedures"[Mesh]) }\end{array}$} & 81 \\
\hline \multicolumn{4}{|c|}{ Pesquisa - CINAHL } \\
\hline 1 & & Checklist & Headings \\
\hline 2 & $A N D$ & Thoracic Surgery & Headings \\
\hline 3 & $O R$ & Thoracic Surgical Procedures & Headings \\
\hline \multicolumn{3}{|c|}{ Estratégia - checklist AND thoracic surgery OR thoracic surgical procedures } & 78 \\
\hline \multicolumn{4}{|c|}{ Pesquisa-SCOPUS } \\
\hline 1 & & Checklist & Palavras chave \\
\hline 2 & $A N D$ & Thoracic Surgery & Palavras chave \\
\hline 4 & $O R$ & Thoracic Surgical Procedures & Palavras chave \\
\hline \multicolumn{4}{|c|}{ Pesquisa - Web of Scienc } \\
\hline \multicolumn{3}{|c|}{ Estratégia - Checklist AND Thoracic Surgery OR Thoracic Surgical Procedures } & 47 \\
\hline 1 & & Checklist & Palavras chave \\
\hline 2 & AND & Thoracic Surgery & Palavras chave \\
\hline \multicolumn{3}{|c|}{ Estratégia - (checklist) AND TOPIC: (thoracic surgery) } & 41 \\
\hline \multicolumn{3}{|c|}{ Pesquisa - Lilacs } & \\
\hline 1 & & Lista de checagem & Decs \\
\hline 2 & OR & Checklist & Decs \\
\hline 3 & AND & Segurança do paciente & Decs \\
\hline 4 & AND & Cirurgia cardiaca & Decs \\
\hline 5 & OR & Cirurgia & Decs \\
\hline \multicolumn{3}{|c|}{$\begin{array}{l}\text { Estratégia - (lista de checagem) or "CHECKLIST" [Palavras] and segurança do } \\
\text { paciente [Palavras] and (cirurgia cardiaca ) or "CIRURGIA" [Palavras] }\end{array}$} & 30 \\
\hline
\end{tabular}

Fonte: Dados da pesquisa (2020).

Para a inclusão dos artigos, foram adotados os seguintes critérios: estudos nos idiomas língua inglês, português e espanhol; artigos com abordagem qualitativa e quantitativa que respondessem à pergunta norteadora. Foram excluídos os artigos duplicados, teses, dissertações e capítulos de livros. Não foi empregado recorte temporal em razão do número limitado de artigos que responderam aos critérios de inclusão.

Utilizou-se o gerenciador de referência na versão gratuita on-line (EndNote) para operacionalizar a seleção dos estudos primários na RI. Após a busca em cada base selecionou-se o filtro de escolha específico da base e realizou-se a importação para o EndNote on-line.

Grupos foram criados dentro do gerenciador de referências para segregação das bases de dados. A ocorrência de estudos duplicados identificou-se pela opção "Find Duplicates" e direcionou para pasta de duplicatas. Após a seleção dos artigos excluídos permaneceram os eleitos segundo o critério de elegibilidade.

Procedeu-se a análise inicialmente pela leitura do título e do resumo das produções, com o intuito de verificar a adequação aos critérios estabelecidos, no segundo momento, houve a leitura na integra das publicações selecionadas. Dois revisores independentes realizaram a pesquisa, no caso de discordância entre os revisores, um terceiro revisor foi consultado e chegado a um consenso. 
Este artigo originou-se do projeto de pesquisa intitulado "Construção e validação de instrumento para a assistência em cirurgia cardíaca segura" aprovado Comitê de Ética em Pesquisa da Universidade Federal de Alagoas, e com base no parecer emitido pelo número 15410219.6.0000.5013.

\section{Resultados}

A busca na literatura de checklist para a cirurgia cardíaca resultou em oito artigos, estes representaram elementos de segurança voltados a especificidade cirúrgica. Quantitativamente não se obteve resposta expressiva em número de artigos, no entanto pode-se perceber uma vasta riqueza de itens apropriados e direcionados a segurança cirúrgica do paciente cardíaco (Figura 1).

Figura 1 - Fluxograma das etapas de seleção duplo-independente (n=08). Maceió, AL, Brasil, 2020.
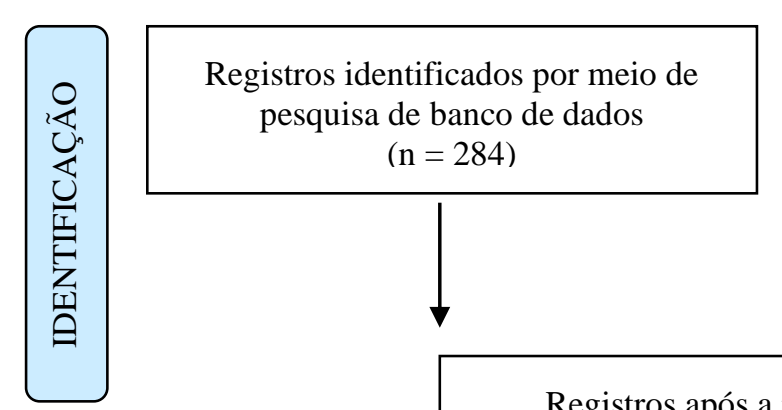

Registros após a remoção de duplicatas $(\mathrm{n}=40)$
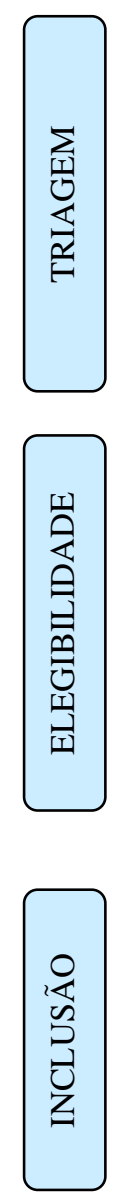

Registros adicionais identificados por outras fontes

$$
(n=0)
$$

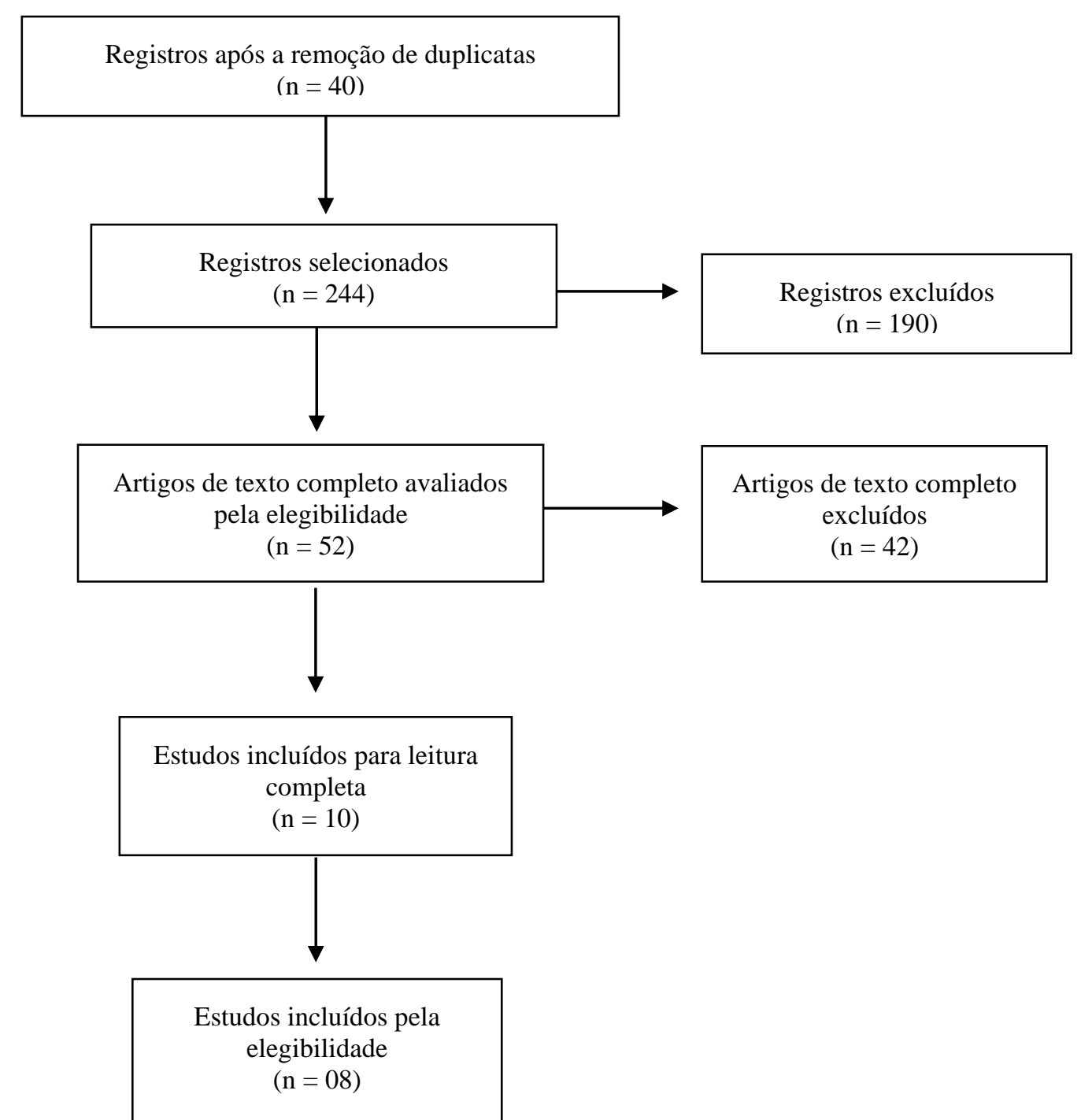

Fonte: Dados da pesquisa (2020). 
Conforme Tabela 1, para cada título de artigo foi direcionado um código, sendo sequenciados entre A1 a A8. No A1 (Berrisford, 2012) observa-se a utilização de uma lista de verificação para reduzir erros de profilaxia de tromboembolismo venoso (TEV) descreve a fase "Time out" relacionando a pergunta do anestesista e a resposta do cirurgião, com ênfase em nove perguntas com o paciente já anestesiado.

O artigo A2 (Hussain et al., 2016). descreve as etapas cruciais envolvidas na inserção de cânulas para procedimentos em cirurgia cardíaca minimamente invasiva, com 22 checagens sequenciadas, há uma lista de verificação que descreve as etapas de inserção de cânulas para procedimentos minimamente invasivos em cirurgia cardíaca. O A3 (Konfirst, 2015) descreve uma lista de verificação desenvolvida para um programa cardíaco pediátrico com direcionamento de checagens para os profissionais, mantendo as fases Sign in, Time out e Sign out, com acréscimo de duas fases pré procedimento, "dia antes da cirurgia e no dia da cirurgia".

No A4 (Ogden et al., 2016) descreve uma lista de verificação para auxiliar na preparação para separação do bypass cardiopulmonar, com nove itens a serem checados. Uma lista de verificação a ser utilizada no período de separação da circulação extracorpórea para decisões, avaliações e pontos de comunicação entre cirurgião, anestesista e perfusionista. Checagens como retorno do ritmo cardíaco normal, necessidade de marcapasso externo, remoção de ar do coração e dos enxertos, necessidade de uso de inotrópicos e vasopressores.

Em A5 (Rakoff, 2018) a lista de verificação de segurança cirúrgica é composta por 44 itens, divididos em cinco fases, modificada localmente. Confirmações de necessidade perfusão seletiva cerebral, colocação de gelo na cabeça e tipo de prime utilizado na circulação extracorpórea foi identificado. O A6 (Raman, 2016) trata-se de uma lista de verificação pré-operatória um modelo de feedback simples com oito questões.

O A7 (Spiess 2013) é subdividido em tabela 1 e 2, a tabela 1 descreve um checklist a ser utilizado pela perfusão com confirmações acerca da identificação do paciente, esterilização dos componentes, máquina coração pulmão, resfriamento do coração, suprimento de gás, bomba, condição da tubulação da bomba inspecionada, checagem da monitorização, segurança e alarmes, oxigenador, tubulação e acessórios. A tabela 2 descreve checagens de cada profissional com inclusão de debriefing ao final do checklist. Aborda conferência da máquina coração pulmão, resfriamento do coração e suplementação de gás anestésico durante a bypass.

Em A8 (Papaspyros, 2010) o controle de glicemia, necessidade de indicação de beta bloqueador e aplicação de segunda dose do antibiótico profilático foram apontadas. Trata-se de um checklist com repostas dicotômicas dividido em processo de etapa geral com quatro itens, Briefing com onze itens e Debriefing com seis itens. 
Tabela 1 - Artigos selecionados conforme código do artigo/ título, fase cirúrgica, profissionais envolvidos, ano/ país e o formato das informações no artigo. Maceió, Al, Brasil, 2020.

\begin{tabular}{|c|c|c|c|c|}
\hline Código artigo / título & País/ Autor/Ano & Fase cirúrgica & $\begin{array}{l}\text { Profissionais } \\
\text { envolvidos }\end{array}$ & Formato \\
\hline $\begin{array}{l}\text { A1 - Surgical time out checklist with } \\
\text { debriefing and multidisciplinary feedback } \\
\text { improves venous thromboembolism } \\
\text { prophylaxis in thoracic surgery: a prospective } \\
\text { audit. }\end{array}$ & $\begin{array}{l}\text { United Kingdom } \\
\text { (UK) } \\
\text { Berrisford et al., } \\
\quad(2011)\end{array}$ & Time out & $\begin{array}{l}\text { Anestesista } \\
\text { Cirurgião }\end{array}$ & $\begin{array}{l}\text { Lista de conferência } \\
\text { no formato de } \\
\text { perguntas }\end{array}$ \\
\hline $\begin{array}{l}\text { A2 - Lessons from aviation - the role of } \\
\text { checklists in minimally invasive cardiac } \\
\text { surgery. }\end{array}$ & $\begin{array}{l}\text { Canadá } \\
\text { Hussain et al., } \\
(2015)\end{array}$ & Time out & $\begin{array}{l}\text { Perfusionista } \\
\text { Cirurgião } \\
\text { Anestesista }\end{array}$ & $\begin{array}{l}\text { Lista de conferência } \\
\text { com checkbox }\end{array}$ \\
\hline $\begin{array}{l}\text { A } 3 \text { - Checklists and Safety in Pediatric Cardiac } \\
\text { Surgery. }\end{array}$ & $\begin{array}{l}\text { United States of } \\
\text { América (USA) } \\
\text { Konfirst et al., } \\
\text { (2015) }\end{array}$ & $\begin{array}{l}\text { Dia antes da } \\
\text { cirurgia } \\
\text { Dia da cirurgia } \\
\text { Sign in (Antes da } \\
\text { indução anestésica) } \\
\text { Time out (Antes da } \\
\text { incisão de pele) } \\
\text { Sign out (Antes da } \\
\text { saída de sala) }\end{array}$ & $\begin{array}{l}\text { Circulante } \\
\text { Anestesista } \\
\text { Cirurgião } \\
\text { Instrumentador }\end{array}$ & $\begin{array}{l}\text { Lista de conferência } \\
\text { com perguntas e } \\
\text { afirmações }\end{array}$ \\
\hline $\begin{array}{l}\text { A4 - Developing a Cardiopulmonay Bypass } \\
\text { Separation Checklist:Consensus Via a } \\
\text { Modified Delphi Technique. }\end{array}$ & $\begin{array}{l}\text { Texas, } \\
\text { United States of } \\
\text { América (USA) } \\
\text { Ogden et al., } \\
\text { (2016) }\end{array}$ & Time out & $\begin{array}{l}\text { Anestesista } \\
\text { Cirurgião } \\
\text { Perfusionista }\end{array}$ & $\begin{array}{l}\text { Lista de conferência } \\
\text { com sequência de } \\
\text { afirmações }\end{array}$ \\
\hline $\begin{array}{l}\text { A5 - Improved Compliance and } \\
\text { Comprehension of a Surgical } \\
\text { Safety Checklist With Customized } \\
\text { Standar } \\
\text { Training: A Randomized Trial. }\end{array}$ & $\begin{array}{l}\text { Índia } \\
\text { Rakoff et al., } \\
\quad(2015)\end{array}$ & $\begin{array}{l}\text { A) Nurse pre-op } \\
\text { verification } \\
\text { checklist } \\
\text { B) } \\
\text { Anesthesia Begins } \\
\text { C) Before Skin } \\
\text { Incision } \\
\text { D) Before Skin } \\
\text { Incision } \\
\text { E) Before Sternal } \\
\text { Closure }\end{array}$ & $\begin{array}{l}\text { Enfermeiro } \\
\text { Cirurgião } \\
\text { Anestesista } \\
\text { Perfusionista }\end{array}$ & $\begin{array}{l}\text { Lista de conferência } \\
\text { com afirmações e } \\
\text { perguntas com } \\
\text { respostas } \\
\text { dicotômicas }\end{array}$ \\
\hline $\begin{array}{l}\text { A6 - When a checklist is not enough: How to } \\
\text { improve them and what else is needed. }\end{array}$ & $\begin{array}{l}\text { Boston, Mass } \\
\text { United States of } \\
\text { América } \\
\text { (USA) } \\
\text { Raman et al., } \\
\text { (2016) }\end{array}$ & Time out & $\begin{array}{l}\text { Cirurgião } \\
\text { Enfermeiro }\end{array}$ & $\begin{array}{l}\text { Lista de conferência } \\
\text { com perguntas }\end{array}$ \\
\hline $\begin{array}{l}\text { A7 - The use of checklist as a method to } \\
\text { reduce human error on cardiac operating } \\
\text { rooms. }\end{array}$ & $\begin{array}{c}\text { Richmond, } \\
\text { Virginia, United } \\
\text { States of América } \\
\text { (USA) } \\
\text { Spiess B. D. (2013) }\end{array}$ & $\begin{array}{l}\text { Tabela } 1 \\
\text { Prebypass checklist } \\
\text { Tabela } 2 \\
\text { Prebtiefing } \\
\text { Postbriefing } \\
\text { Debriefing }\end{array}$ & $\begin{array}{l}\text { Tabela } \\
\text { Perfusionista } \\
\text { Tabela } 2 \text { Cirurgião, } \\
\text { Enfermeiro, } \\
\text { Anestesista, } \\
\text { Perfusionista }\end{array}$ & $\begin{array}{l}\text { Lista de conferência } \\
\text { com sequência de } \\
\text { afirmações }\end{array}$ \\
\hline $\begin{array}{l}\text { A8 - Briefing and debriefing in the cardiac } \\
\text { operating room. Analysis of impact on theatre } \\
\text { team attitude and patient safety. }\end{array}$ & $\begin{array}{l}\text { United Kingdom } \\
\qquad(\mathrm{UK}) \\
\text { Papaspyros } \\
\text { et al., (2010) }\end{array}$ & $\begin{array}{l}\text { General Step } \\
\text { process } \\
\text { Briefing }- \text { before } \\
\text { every procedure } \\
\text { Debriefing }- \text { After } \\
\text { every procedure }\end{array}$ & $\begin{array}{l}\text { Anestesista } \\
\text { Cirurgião } \\
\text { Circulante } \\
\text { Perfusionista }\end{array}$ & $\begin{array}{l}\text { Lista de conferência } \\
\text { com perguntas e } \\
\text { respostas } \\
\text { dicotômicas }\end{array}$ \\
\hline
\end{tabular}

Fonte: Dados da pesquisa (2020).

\section{Discussão}

Com base nos estudos da presente revisão integrativa os artigos apontaram diferentes formatos e maneiras de fazer verificações, identificou-se subespecificações de checagens, abrangendo assuntos da cirurgia cardíaca. Importante ressaltar que os componentes coloquialmente conhecidos "Sign in, Time out e Sign out" permanecem como componentes chaves da 
lista de verificação, reafirmando o recomendado pela OMS em 2009 (Tan et al., 2021). Os artigos A3 e A5 utilizaram a formatação recomendada pela OMS com acréscimo de uma etapa antes do dia da cirurgia. A1, A2, A4 e A6 descreveram a fase "Time out" e A7 e A8 descreveram as fases Briefing e Debriefing.

De modo geral, as checagens permitem auxiliar a equipe na confirmação de situações de rotina ou de emergência, para que melhorem a comunicação por meio de modelos mentais compartilhados e não se esqueçam de itens essenciais. Em situações de emergência lista de verificação é altamente eficaz e representa um esforço coordenado da equipe, exigindo que várias pessoas capacitadas se comuniquem e tomem as devidas providências (Spiess 2013).

Um ponto em comum descrito nos artigos é que a lista de verificação por si só não trás os resultados esperados, A1 descreve "a lista é apenas um elemento, o importante é uma cultura de segurança aprimorada", A2 "embora as listas de verificação orientem os usuários, elas não resolvem todos os problemas", A3 "a lista de verificação é um processo facilitador", A4 "lista de verificação defeituosa pode aumentar o risco do paciente", A5 "as diferenças culturais podem impedir a implementação eficaz do checklist", A6 "o uso excessivo de lista de verificação sem fazer mudanças no sistema está começando a ser reconhecido na área da saúde", A7 "se houver uma cultura de segurança e as listas de verificação forem projetadas corretamente, os usuários poderão abraçar a lista e descobrir que, em vez de alongar, ela encurta a carga de trabalho", A8 "o conceito de briefing/ debriefing adiciona objetivos educacionais para promover a segurança do paciente". Com isso o conhecimento de como implementar intervenções evidenciadas de forma eficaz e sustentável em escala permanente é o foco da ciência da segurança do paciente (White et al., 2021).

É importante que a equipe cirúrgica continue aperfeiçoando o nível de compreensão acerca de técnicas mais eficazes baseadas em evidências para um atendimento mais seguro ao paciente cardíaco cirúrgico e um cuidado livre de riscos e danos. Estudo publicado descreve mal-entendido entre cirurgião e perfusionista durante o desmame da circulação extracorpórea (CEC), durante o debriefing a equipe reconheceu que duas conversas de fundo impediram o cirurgião e o perfusionista de se comunicarem claramente (Papaspyros et al., 2010). Outro estudo relata incidentes em procedimentos cardíacos por distintos enfermeiros, afirmando a necessidade de direcionamento e capacitação da equipe para a especialidade (Raman et al., 2016).

Nesta perspectiva, a adesão aos instrumentos como checklists para o cotidiano da assistência pode sinalizar potenciais riscos, servir de barreiras para a quebra da segurança, bem como gerar ciclo de melhoria que busque a prestação de assistência livre de danos (Amaya et al., 2016). É primordial para a segurança do paciente e da equipe que protocolos sejam instituídos e incorporado no dia a dia dos serviços de saúde, evitando danos e prejuízos à saúde da pessoa que está sendo submetida a um procedimento cirúrgico, além de garantir mais autonomia e proteção ao profissional e à instituição como um todo (Bernardo et al., 2021).

\section{Conclusão}

Neste estudo foi possível constatar na literatura a existência de checklist para cirurgia cardíaca segura, evidências que reúnem elementos de verificação com informações objetivas para a equipe cirúrgica e de enfermagem realizar ações básicas de segurança capaz de mitigar a ocorrência de eventos adversos, se implementado e utilizado adequadamente. Contribuindo para reflexão sobre as práticas no contexto da cirurgia cardíaca.

Desse modo, recomenda-se a utilização dos checklists deste estudo para a segurança do paciente em cirurgia cardíaca como modelo para as mais diversas práticas locais pois atua como lembrete de etapas simples que favorece o trabalho em equipe e a melhora a comunicação. Além do desenvolvimento de outros estudos entre diferentes contextos para a segurança do paciente cardíaco.

Como limitação desta revisão está a dificuldade em encontrar artigos mais atuais e que trouxesse informações de eficácia da utilização, melhoria nos processos e resultados para os pacientes. 


\section{Referências}

Amaya, M. R., Paixão, D. P. S., Sarquis, L. M. M., \& Cruz, E. D. A. (2016). Construção e validação de conteúdo de checklist para a segurança do paciente em emergência. Rev Gaúcha Enferm 37(spe): e68778.

Bernardo, T. H., Silva, I, M, F., Lima, S. T., Thomé, A. R. C. S., Soares, I. P., Bernardo, R. C. C., Santos, S. S. G. S. (2021). Identificação dos requisitos necessários para um checklist de cirurgia cardíaca segura. Research Society and Development 10 (4).

Berrisford, R. G., wilson, I. H., Davidge, M., \& Sanders, S. (2012). Surgical time out checklist with debriefing and multidisciplinary feedback improves venous thromboembolism prophylaxis in thoracic surgery: a prospective audit. Eur j cardiothorac surg. 41(6),1326-1329.

Chan, J., Gupta, A. K., Stewart, S., Babidge, W., Mc Culloch, G., Worthington, M. G., \& Maddern, G. J. (2019). "Nobody told me": Communication Issues Affecting Australian Cardiothoracic Surgery Patients. The Annals of thoracic surgery, 108(6), 1801-1806.

Earnshaw, J. J., \& Alderson, D. (2014). Sustainable global surgery. British journal of surgery 101(2), 1-2.

Hussain, S., Adams, C., Cleland, A., Jones, P., Walsh, G., \& Kiaii. B. (2016). Lessons from aviation - the role of checklists in minimally invasive cardiac surgery. Perfusion 31(1), 68-71.

Joanna Briggs Institute. (2014). Reviewers Manual-Methodology for JBI Mixed Methods Systematic Reviews.

Kim, F. J., da Silva, R. D., Gustafson, D., Nogueira, L., Harlin, T., \& Paul, D. L. (2015). Current issues in patient safety in surgery: a review. Patient Saf Surg 9 (26).

Konfirst, C., Preston, S., \& Yeh, Jr. T. (2015). Checklists and safety in pediatric cardiac surgery. Semin Thorac Cardiovasc Surg Pediatr Card Surg Annu $18(1), 43-50$.

Liukka, M., Steven, A., Moreno, M., Sara-Aho, A. M., Khakurel, J., Pearson, P., Turunen, H., \& Tella, S. (2020). Action after Adverse Events in Healthcare: An Integrative Literature Review. International journal of environmental research and public health, 17(13), 4717.

Mendes, K. D. S., Silveira, R. C.C. P., \& Galvão, C. M. (2019). Uso de gerenciador de referências bibliográficas na seleção dos estudos primários em revisão integrativa. Texto contexto - enferm. 28:E20170204.

Ogden, S. R., Culp, Jr. W. C., Villamaria, F. J., \& Ball, T. R. (2016). Developing a Checklist: Consensus Via a Modified Delphi. Technique J Cardiothorac Vasc Anesth 30(4), 855-858.

Organização Mundial da Saúde. (2009). Segundo desafio global para a segurança do paciente: Cirurgias seguras salvam vidas. Organização Pan-Americana da Saúde/Ministério da Saúde/Agência Nacional de Vigilância Sanitária.

Papaspyros, S. C., Javangula, K. C., \& Adluri, R. K. P. (2010). O'Regan DJ. Briefing and debriefing in the cardiac operating room. Analysis of impact on theatre team attitude and patient safety. Interact Cardiovasc Thorac Surg 10 (1), 43-47.

Rakoff, D., Akella, K., Guruvegowda, C., Chhajwani, S., Seshadri, S., \& Sola, S. (2018). Improved Compliance and Comprehension of a Surgical Safety Checklist With Customized Versus Standard Training: A Randomized Trial. J Patient Saf. 14 (3), 138-142.

Raman, J., Leveson, N., Samost, A. L., Dobrilovic, N., Oldham, M., Dekker, S., \& Finkelstein., Stan (2016). When a checklist is not enough: How to improve them and what else is needed. J Thorac Cardiovasc Surg 152 (2), 585-592.

Russ, S., Rout, S., Caris, J., Mansell, J., Davies, R., Mayer, E., Moorthy, K., Darzi, Ara., Vicent, C., \& Sevdalis, N. (2015). Measuring variation in use of the Measuring variation in use of the WHO surgical safety checklist in the operating room: a multicenter prospective cross-sectional study. $J$ Am Coll Surg. 220 (1), 1-11.

Spiess, B. D. (2013). The use of checklists as a method to reduce human error in cardiac operating rooms. Int Anesthesiol Clin. 51(1), $179-194$.

Tan, J., Ngwayi, J. R. M., Ding, Z., Zhou, Y., Li, M., Chen, Y., Hu, Bingtao., Liu, J., \& Porter, D. (2021). Attitudes and compliance with the WHO surgical safety checklist: a survey among surgeons and operating room staff in 138 hospitals in China. Patient Saf Surg 15 (3).

Thomé, A. R. C. S., Melo, E. S., Silva, V. M. S., Almeida, T. G., Farias, I. P. \& Vasconcelos, E. L. (2017). Construção e validação de instrumento para assistência em cirurgia cardíaca segura. Revista de enfermagem UFPE on line.

White, M. C., Peven, K., Clancy, O., Okonkwo, I., Bakolis, I., Russ, S., Leather, A., \& Sevdalis, N. (2021). Implementation Strategies and the Uptake of the World Health Organization Surgical Safety Checklist in Low and Middle Income Countries: A Systematic Review and Meta-analysis. Annals of surgery, 273(6), e196-e205.

World Health Organization. (2019). World Patient Safety Day. 